\title{
CONTRIBUTORS TO THE ISSUE
}

Aguilar, Calista

Anasuyamma, $U$.

Aoshima, Kengo

Arnold, David

Banik, Bimal K.

Blythe, Eric

Boyle, Peter H.

Burtea, Carmen

Dalloul, Hany M.

Dandala, Ramesh

Dilmaghani, Karim Akbari

Elst, L. Vander

Farrell, Martilias S.

Garcia, Isabella

Harak, S. S.

Haranath, P.

Hasan, Aurangzeb

Jain, M. S.

Kamikage, Nao

Kato, Tatsunori

Khan, Sadaf Sadiq

Kobayashi, Masatsugu

Kumar, B. Siva

Laurent, Sofie

$\mathrm{Li}$, Jianyong

Li, Weisi

Li, Zhenjiang

Medved, Miroslav

mirzaei, Mansoor

Morales, Frances R.

Muller, Robert N.

Naidu, Andra

Önal, Zülbiye

Oshikawa, Tatsuo

Ouyang, Pingkai
109 Patil, B. P.

101 Rao, Divvela V.N. Srinivasa 121

97 Reddy, C. Suresh 147

109 Ren, Xinghua 125

97 Rosa, Michael De 97

155 Sakahara, Harumi 101

101 Seals, Tamika 97

155 Shi, Yuhu 125

121 Sun, Yingjie 125

139 Takahashi, Masaki 101

101 Takayanagi, Hisao 101

97 Takehara, Yasuo 101

109 Trinadhachari, Ganala Naga 121

165 Tsolomiti, Georgia 143

147 Tsolomiti, Georgia 161

131 Tsolomiti, Georgia 173

165 Tsolomiti, Kyriaki 143

101 Tsolomiti, Kyriaki 161

101 Tsolomiti, Kyriaki 173

131 Tsolomitis, Athanase 143

101 Tsolomitis, Athanase 161

147 Tsolomitis, Athanase 173

101 Wagh, B. S. 165

183 Wagh, S. B. 165

125 Wills, Kristin 97

125 Y Bharathi Kumari 177

97 Yamashita, Mitsuji 101

139 Yıldırım, Ismail 113

109 Yu, Gang $\quad 101$

101 Zeynizadeh, Behzad 139

121 Zhang, Jiyu 183

113 Zhang, Ping 183

101 Zhou, Xu-zheng 183 